\title{
Digital chaos synchronization in optical networks
}

\author{
A. González-Marcos and J.A. Martín-Pereda
}

ETS Ingenieros de Telecomunicación, Universidad Politécnica de Madrid

Ciudad Universitaria s/n. 28040 - Madrid, Spain

Telephone: +34.1.336.73.04, Fax:+34.1.336.73.19

jamp@tfo.upm.es;agonmar@tfo.upm.es

\begin{abstract}
A new method to obtain digital chaos synchronization between two systems is reported. It is based on the use of Optically Programmable Logic Cells as chaos generators. When these cells are feedbacked, periodic and chaotic behaviours are obtained. They depend on the ratio between internal and external delay times. Chaos synchronization is obtained if a common driving signal feeds both systems. A control to impose the same boundary conditions to both systems is added to the emitter. New techniques to analyse digital chaos are presented. The main application of these structures is to obtain secure communications in optical networks.
\end{abstract}

Keywords

Chaos syncronization, secure communications, digital chaos.

\section{INTRODUCTION}

The original version of this chapter was revised: The copyright line was incorrect. This has been corrected. The Erratum to this chapter is available at DOI: 10.1007/978-0-387-35398-2_19 


\section{Part Seven: Advanced Topics in optical networks}

A considerable interest has appeared in the last years concerning the application of chaotic circuits in order to obtain secure communications. The broad-band nature of chaotic signals makes them tempting for use in this area and in spread-spectrum situations. The fact that such signals emanate from a deterministic dynamic system lends to the hope that one will also be able to control them sufficiently for many uses. Taken as isolated systems, chaotic dynamics appears to offer many impediments to anyone attempting to put them to use. The main problem to be addressed is then how to synchronize chaotic circuits. It is well known that two identical circuits are able to offer similar chaotic outputs. But if these circuits are not synchronized their output signals are not valid to be employed in a communication system. The main reason is the strong dependence of the obtained chaos on the initial and boundary conditions of the chaos generator circuit. Two chaotic signals with the same characteristics may have different values at any particular time if they are generated independently. Some additional conditions have to be imposed to the system in order to obtain identical chaotic signals at any time. One of these conditions is the synchronization.

Several attempts have been made in this direction. The idea that chaotic systems could synchronize was first put forth in a paper more than ten years ago (Afraimovich, 1986). Several authors have followed the lines indicated in that paper. Pecora (1990) and Carroll (1991) demonstrated the possibility of sinchronizing chaotic subsystems with a common driving signal. Their idea was to decompose the chaotic dynamical system in two subsystems, "driving" and response" subsystems. The driving subsystem is composed by two state variable components whereas the second one just has one and uses as input signal one of the state components of the first subsystem. Some examples following this idea and schemes, using Chua's circuits, are reported in the literature (Madan, 1993).

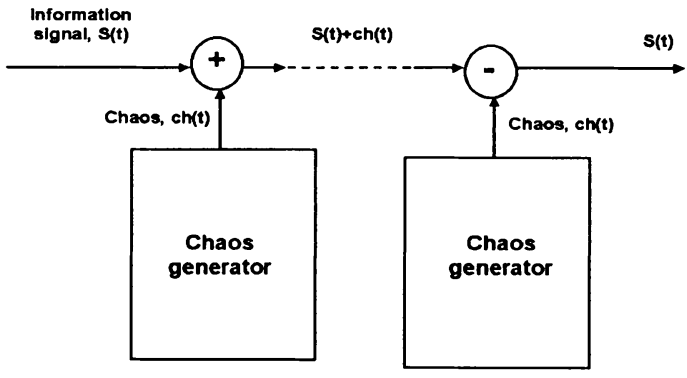

Figure 1 Basic configuration of the communication system

Another additional point needs to be considered. It concerns the characteristics of the chaotic signal to be employed. Almost in any of the reported situations the obtained chaos is analog. Although all physical systems are really analog, communication and computer systems are nowadays digital. The way to use analog signals in digital systems in to make the conversion analog/digital. This idea has been employed in any case where an application to communication is needed. It should be useful to obtain digital chaos from the very beginning of the process and to employ it with the same requirements of the information signals. 
The purpose of this paper is to present a way to obtain digital chaos and how to synchronize two chaotic systems. The scheme of the proposed system is shown in Fig. 1. Two identical chaos generators, A and B, are located at emitter and receiver. Information signal is added at the receiver and transmitted to the network. This composed signal is detected at the receiver and processed with another chaotic signal obtained there. The resulting signal is the information generated at the emitter. This configuration is the basis of our system.

\section{OPTICAL PROGRAMMABLE LOGIC CELL (OPLC)}

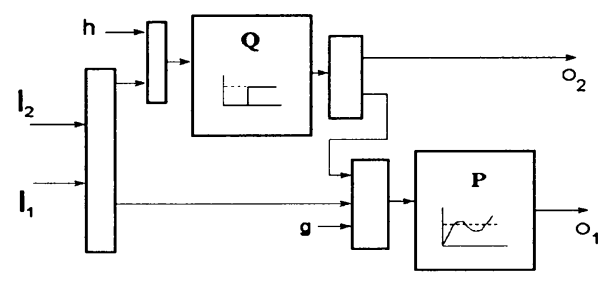

Figure 2 Internal configuration of an OPLC.

The main block of our chaos generator is an Optically Programmable Logic Cell employed previously by us as a part of a possible optical computer (Martín-Pereda, 1992). Although this structure has been reported in several places, some of its principal characteristics will be here presented again. Its main characteristic is the logic processing of two input binary signals, governed by two control signals. Two outputs give logical functions of these inputs. The type of processing is related to the eight main Boolean Functions, namely, AND, OR, XOR, NAND, NOR, XNOR, ON and OFF. The programmable ability of the two outputs, as it has been described, allows the generation of several data-coding for optical transmission. Moreover, as it was shown, this circuit has the possibility to the generation of periodic and even chaotic solutions. A precise analysis of the output characteristic versus the main variable parameters, as control signal level and data signal level, has been reported (González-Marcos, 1995).

With this configuration, the above mentioned digital character of the signal is directly obtained. Its main blocks are shown in Fig. 2. Two devices with a non-linear behaviour, $\mathrm{P}$ and $\mathrm{Q}$, compose the circuit. The outputs of each one of them correspond to the two final outputs, $\mathrm{O}_{1}$ and $\mathrm{O}_{2}$, of the cell. Four are the possible inputs to the circuit. Two of them are for input data, $\mathbf{I}_{1}$ e $\mathbf{I}_{2}$, and the other two, $g$ and $h$, for control signals. The way these four inputs are arranged inside the circuit, is also represented in Figure 2. A practical implementation we have carried out of the processing element has been based on an optoelectronical configuration. Lines in Fig. 2 represent optical multimode fibers. The indicated blocks, placed in order to combine the corresponding signals, are conventional optical couplers. In this way, optical inputs arriving to the individual devices are multilevel signals. The characteristics of the non-linear devices are also shown in Fig. 2. Device Q, corresponds to a thresholding or switching 
device, and device $\mathrm{P}$ is a multistate device, being the response of this non-linear optical device the one represented in Fig. 2. This response is similar to the behaviour of a SEED device (Miller, 1985).

\section{CHAOS GENERATION FROM AN OPLC}

A non-linear behaviour is expected if some kind of feedback is applied to this cell (Ikeda, 1979), (Okada, 1981), (Neyer, 1982). The feedback we have applied to the system, among the different possibilities, is the one going from the output $\mathrm{O}_{1}$ of $\mathrm{P}$ device (see Fig. 3) to the control input, $g$, of P-device. No other additional control signal has been used. A chaotic output is obtained when the internal response time is made equal to zero or is much smaller than the external one. Some results have been reported by us (González-Marcos, 1996).

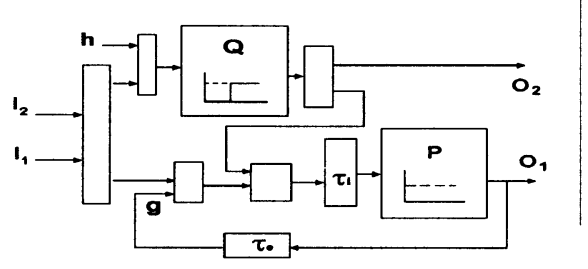

Figure 3 Optical Programmable Logic Cell with feedback. Chaos Generator.

In order to characterize the obtained chaotic signal, conventional methods are difficult to be applied here. This is due to its digital character (Martín-Pereda, 1995b). The results constitute a Time Series from where a chaos measure should be obtained. But the correct phase-space representation is not possible to be grasped from these results in a straightforward way. We do not even know what the adequate phase-space variables are and it is not even known how many variables are needed to fully describe the dynamics of this particular system. There is fortunately a partial answer to this problem that has been applied successfully in a large number of experimental investigations. The basic idea is that if the fundamental phase-space variables are $\mathbf{x}$ and $\mathbf{x}$ ', to study the evolution of the system numerically, $x$ and $x^{\prime}$ have to be follow as functions of $t$. But since $x^{\prime}=[x(t+\Delta t)-x(t)] / \Delta t$ in the limit as $\Delta t \rightarrow 0$, a knowledge of $x(t+\Delta t)$ is equivalent to a knowledge of $x^{\prime}$. In other words, a knowledge of a trajectory of points $[x(t), x(t+\Delta t)]$ is equivalent to a knowledge of the trajectory of points $\left[x(t), x^{\prime}(t)\right]$. As a consequence, a phase-space trajectory

$x(t)=\left[x_{1}(t), x_{2}(t), \ldots, x_{n}(t)\right]$

is replaced by a trajectory in an artificial phase space with points given by

$y(t)=[y(t), y(t+\Delta t), \ldots, y(t+m \Delta t)]$ 
where $y(t)$ is any one of the phase-space variables $x_{i}(t)$. Thus from a set of measurements of a single quantity $y(t)$ we can construct a sequence of points in an artificial phase space

$x(t)=[y(t), y(t+\Delta t), \ldots, y(t+m \Delta t)]$

$x(t+\Delta t)=\{y(t+\Delta t), \ldots, y[t+(m+1) \Delta t]\}$

With the data we have, the first problem to solve is how to operate with our digital signal where just two values, "0" and "1", are present. If we adopt just this output as possible values for $\mathbf{y}$, the resulting plot at the phase space should be concentrated on just four points, namely, $(0,0),(0,1),(1,0)$ and $(1,1)$. Almost no information could be obtained from it. Hence a new technique has to be implemented.

The method we have adopted is to group sets of four consecutive bits and to convert them to their corresponding hexadecimal values. Hence, a sequence of zeroes and ones is converted to a new string of hexadecimal values, namely, $0,1,2, \ldots . ., 15$. For example, "0010" would be a "2", "1001" a "9" and "1110" a "14". The total number of data is divided by four, but much more information can be obtained from them than with simple binary signals. A diagram, similar to the $t_{i+1}$ versus $t_{i}$ in analogue signals, can be drawn in this way. In the case of periodic signals, a closed configuration is obtained. But in the case of chaotic signals, no definite pattern would be obtained. Some results are reported in González-Marcos (1996).

A further point needs to be considered. It is the one concerning the justification that the preceding quantity, namely the hexadecimal sequence, represents the same behavior of the system than the previously obtained binary one. But this situation is equivalent to the reverse one: to convert a chaotic analog signal into a digital one. As it is well known from digital communications, any analog signal can be quantized and from this quantization to obtain a digital signal with the same main properties than the initial analog one. In our present case, we have accomplished the opposite operation, namely, to convert a digital signal into an analog quantized one with sixteen possible levels. And, according to digital communication signal processing theory, both representations are equivalent. 


\section{SYNCHRONIZATION OF CHAOTIC OPLCS}

If two identical cells with feedback, as the above mentioned, are parallel connected (Fig. 4) and the same signal arrives to their inputs, an identical chaos is obtained at their outputs. This situation corresponds to two identical and ideal configurations working under identical conditions.

The behaviour becomes critical when the simulation tries to be close to a real situation. In this case, if both systems are not feed by exactly the same signal, the obtained outputs, although chaotic, are different. Hence, no possible relation between then should be feasible.

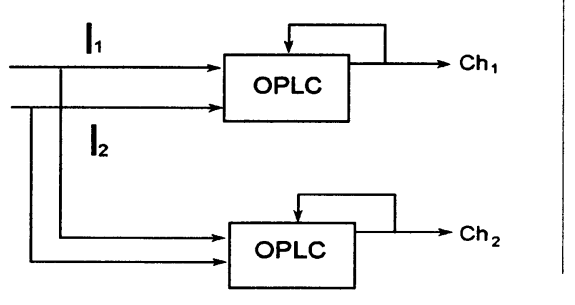

Figure 4 Basic blocks for chaos syncronization

In a general situation, both systems, emitter and receiver, are located at different places. As a consequence, there is no possibility to introduce exactly the same input signals to their corresponding input ports. This is because although a common signal generator could send the same train of pulses to both cells, the arriving times to them can be different. The time need to get the first cell is known if this generator is at the receiver place. But the time when the signal arrives to the second cell may not be known. This is the most general case. Several solutions could be implemented to overcome this fact. The solution we have adopted is presented in Fig. 5, and it was partially reported previously (Martín-Pereda, 1996). 


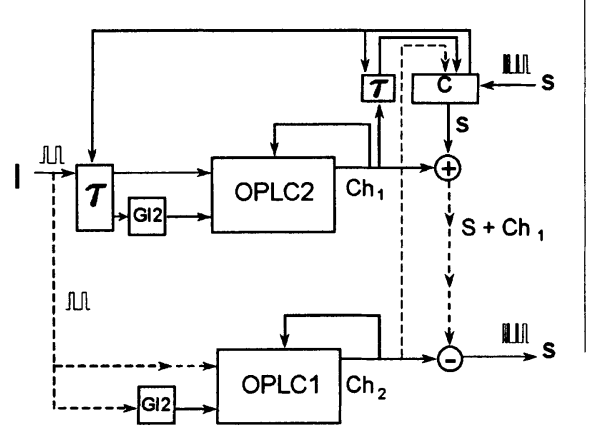

Figure 5 Schematic diagram of the communication system

The same driving signal is sent to both cells. A delay time, $\tau$, is added before this signal gets into the emitter cell. This time has to be equal to the fly time from emitter to receiver. But this time is not known. Hence has to be changed until both times are the same. The way to change is imposed by the signal given by comparator $\mathrm{C}$. There, the chaos signal coming from OPLC2 is compared with the chaotic signal obtained from OPLC1 and depending on the difference between these two signals, the order is given. As before, a delay time $\tau$ is added to the output from the OPLC1.

Two methods have been implemented in order to know the time delay magnitude to be added at the receiver. The first one is shown in Fig. 6.a. Chaos signal from OPLC1 is represented at the $\mathrm{x}$ axis and the one from OPLC2 at the $\mathrm{y}$ axis. A hexadecimal representation, as before, was taken. The second method is just to represent the difference "Chaos from OPLC1 - Chaos from OPLC2)" versus time. An example is given at Fig. 6.b.

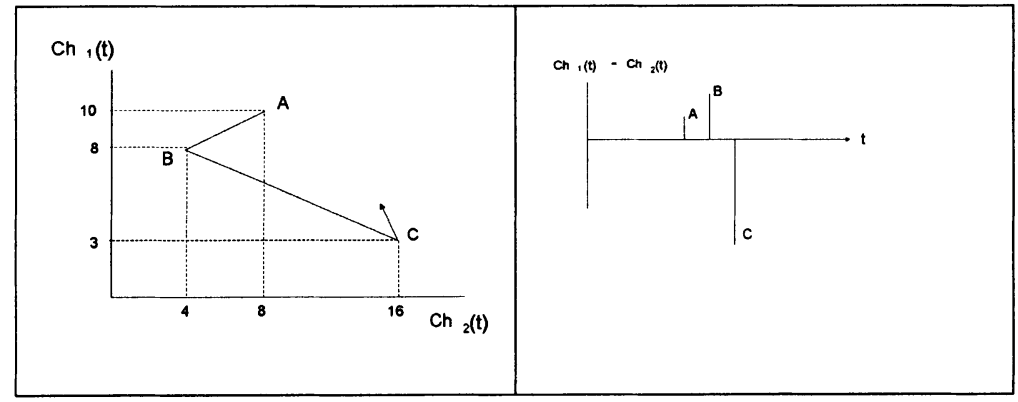

Figure 6.a

Figure 6.b 
Another point needs to be considered. Because the strong dependence with respect to the initial conditions, it is necessary to reset to zero every time the delay time is changed. The variation should follow a line like the one represented in Fig. 7.

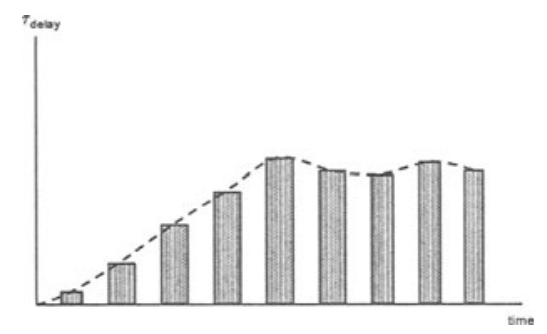

Figure 7 Delay time evolution to syncronize chaos generators en figure 5.

Some particular cases are represented in Fig. 8.a-d. A constant delay time equal to 20 time units has been taken in every case. The change between different representations is in the internal delay time added. An interesting fact needs to be pointed out. As it is seen, the main characteristics of the obtained graph, when delay time is smaller than fly time, is equal to the obtained when is larger by the same amount, but with a different angle with respect to the co-ordinate axis. This gives us an indication of the situation of the guessed delay time imposed to OPLC1. A similar result, but just with an indication of the chaos difference magnitude, is obtained from the above-mentioned second method.

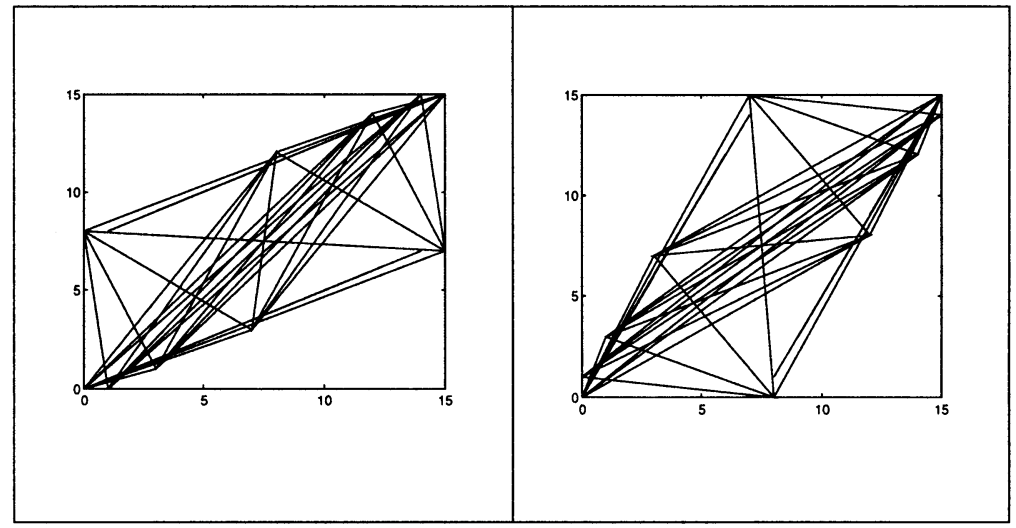

Figure 8.a Time delay $=19$

Figure 8.b Time delay $=21$ 


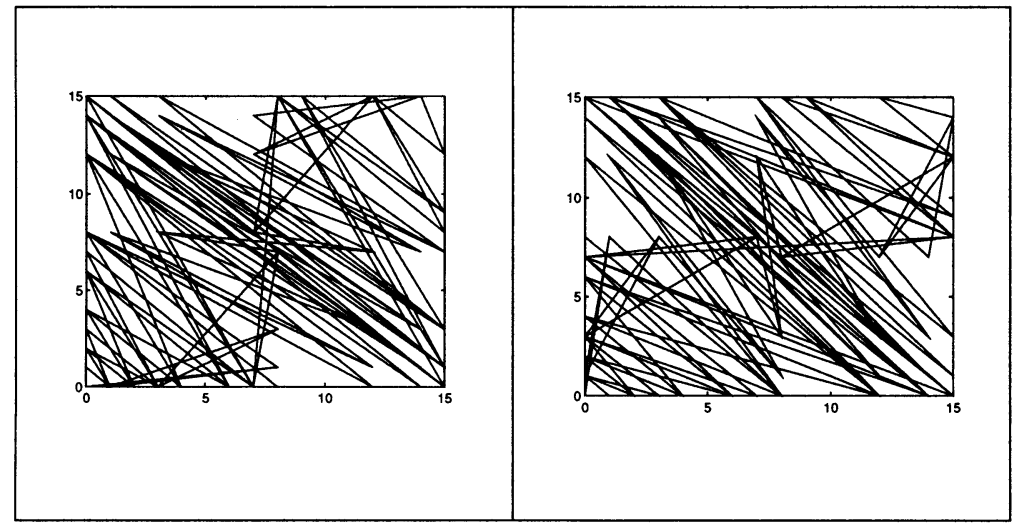

Figure 8.c Time delay $=25$

Figure 8.d Time delay $=15$

The final result, for a fly time of 20 time units and an initial imposed delay time of 15 is shown in Fig. 9. It shows "Chaos from OPLC1 minus chaos from OPLC2" versus time. As it can be seen, after a certain number of changes in delay time, synchronization is obtained.

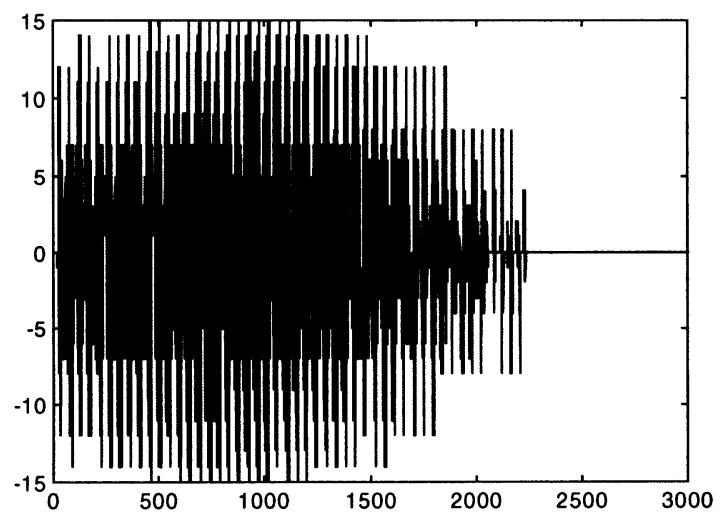

Figure 6 Substration of chaos signals from both channels. 


\section{CONCLUSIONS}

A new method to synchronize chaotic circuits has been reported. The method is based on the use of Optically Programmable Logic Cells as chaos generators. Although these cells have been studied previously, some further studies are needed in order to determine the characteristics of the obtained chaos when the initial driving signal changes. The dependence with its parameters deserves also a deeper study. Moreover, the added delay time has been set to a certain value and if it is not the adequate one, the cell is reset to zero and the process start again. An study of how small and continuos changes affect the results are needed.

Finally, another point should be considered. It concerns the transfer of information among biological systems as well as their behaviour. Some pathologies and malfunctions in living bodies can be analyzed with similar methods as the above reported (Martín-Pereda, 1995 a). This area deserves a further study in order to approach these two fields, communication systems and communication between neurons.

\section{Acknowledgement}

This work has been partially supported by the Community of Madrid under research project 07T/0017/1997.

\section{REFERENCES}

Afraimovich, V.S.; Verichev N.N. and Rabinovich, M.I. (1986) Stochastic synchronization of oscillations in dissipative systems, Inv. VUZ. Rasiofiz. RPQAEC 29, 795-803, 1986.

Carroll, T.L. and Pecora, L.M. (1991) Synchronizing Chaotic Circuits, IEEE Trans. on Circuits and Systems, 38, 453.

González-Marcos, A. and Martín-Pereda, J.A. (1995) Sequences of bifurcations and transition to Chaos in an Optically-Processing Element, in Optical Computing (ed. B..S. Wherrett), . Inst. Phys. Conf. Series. No. 139 Part IV.

González-Marcos, A. and Martín-Pereda, J.A. (1996) Digital Chaotic Output from an OpticallyProcessing Element. Optical Engineering, 35, 525-535. 
Ikeda K. (1979) Multiple-valued stationary state and its instability of the transmitted light by a ring cavity system. Optical Communication, 30, 257-261.

Madan (1993), in Chua's Circuit: A Paradigm for Chaos (ed. R.N. Madan) World Scientific Series on Nonlinear Science. World Scientific. London.

Martín-Pereda, J.A. and González-Marcos, A. (1992) Optical Programmable Processing Element using Optical Fibers, IEEE Lasers and Electro-Optics Society, LEOS'92. Boston, 15-20 November.

Martín-Pereda, J.A. and González-Marcos, A.(1995a) Some Connections between Neurophysiology and Optical Computing based on the Theory of Complexity, in Fluctuation Phenomena: Disorder and Nonlinarity (ed. J. Bishop \& L. Vázquez), World Scientific Press, Singapur.

Martín-Pereda J.A. and González-Marcos A. (1995b) Digital chaos analysis in optical logic structures. SPIE's Photonics East'95 Symposium. Philadelphia, 22-26.

Martín-Pereda J.A., González-Marcos A. and Sánchez-Guillén C. (1996) Synchronizing Chaotic Optically-Programmable Digital Circuits. Globecom 96. IEEE Global Telecommunications Conference, Londres.

Miller, David A. B., Daniel S. Chemla, Theodore C. Damen, Thomas H. Wood, Charles A. Burrus Jr, Arthur C. Gossard and William Wiegmann, (1985) The Quantum Well Self- Electrooptic Effect Device: Optoelectronic Bistability and Oscillation, and Self-Linearized Modulation, IEEE J. of Quantum Electronics, QE-21, No. 9, 1462-1476.

Neyer A. and Voges E. (1982) Dynamics of Electrooptic Bistable Devices with Delayed Feedback. IEEE J. Quantum Electronics, QE-18, 2009-2015.

Okada M. and Takizawa K. (1981) Instability of an electrooptic bistable device with a delayed feedback. IEEE J. Quantum Electronics, QE-17, 2135-2140.

Pecora, L.M. and Carroll, T.L. (1990) Synchronization in Chaotic Systems, Physical Review Letters, 64, 821 .

Pecora, L.M. (1993) Overview of Chaos and Communications Research, in "Chaos in Communications", SPIE Proceedings 2038, 2-25. Bellingham, WA.

\section{BIOGRAPHY}

A. GONZÁLEZ-MARCOS She got her Ph.D. from ETS. Ingenieros de Telecomunicacion, (U.P.M.) Madrid Politechnical University, in 1993. She was working previously for some private companies in CAD-CAE topics. After her Doctoral Thesis, she was Assistant Professor at the Alcalá de Henares University and Associate Professor at the Alfonso X University, both in Madrid. She is presently Professor, from 1996, at the Technical Higher School of Telecommunication Engineers, U.P.M. Her present interest is on Optical Computing, on the applications of Chaos Theory to Optical Communications and on the modelling of the mammalian retina. She has published several papers on the above topics in technical journals. She is member of several technical associations. 
186 Part Seven: Advanced Topics in optical networks

J. A. MARTIN-PEREDA He got his Ph.D. from ETS. Ingenieros de Telecomunicación in 1971. He is full Professor at the Madrid Politechnical University from 1975 and member of several Technical Programme Conferences, mainly in Optical Communications (ECOC, OFC, IOOC) since 1985. Member of the ESPRIT Advisory Board between 1990 and 1993. Member of the Royal Spanish Academy of Engineering since 1995. Senior Member of IEEE and Member of OSA and SPIE. He has published more than 100 papers in journals and books and has got several academic and technical prizes. His present interest is on the application to Chaos Theory to Optical Communications and Biological Systems. 